\title{
Practical Experiences on a Road Guidance Protocol for Intersection Collision Warning Application
}

\author{
Hyun Jeong Yun*, Jeong Dan Choi* \\ *Cooperative Vehicle-Infra Research Section, ETRI, 138 Gajeong-ro Yuseong-gu, KOREA \\ hiyun@etri.re.kr, jdchoi@etri.re.kr
}

\begin{abstract}
In this paper, we provide an overview on a standardization activity in ISO TC204 WG17 for ISO 13184 series and discuss how to develop the Road Guidance Protocol (ISO 13184-2) and deploy it for the road safety use cases implementation based on V2X using the Personal ITS-Station. We have a standardization activity to specify the protocol which is designed to be flexible and extendable, which enables to add or delete Cooperative ITS use cases conveniently. Also we had a field trial of the RGP applied CAVG (Cooperative Autonomous Vehicle Guidance) system at a three-legged intersection as a test site and verified the RGP between the VGSS (Vehicle Guidance Server Subsystem) and the VGTS (Vehicle Guidance Terminal Subsystem) to periodically exchanges information (e.g., position, moving speed, direction, collision-possible and so on) using WAVE/DSRC technology.
\end{abstract}

Keywords - Road Guidance, Data eXchange Message, CALM, Intersection Collision, Road Safety, Cooperative ITS

\section{INTRODUCTION}

Modern vehicles equipped with driver assistance systems can be driven either manually or autonomously. These vehicles can "feel" with sensors, can "see" with cameras and can "speak" with V2X communication devices. V2X communication technology allows for dynamic real-time information to increase driver safety and traffic efficiency, and to enable the collection of valuable data for environmental purposes. V2X technology is one core solution in the "Cooperative ITS" environment. Cooperative means that the ITS station involves both road side, vehicle side and personal side working together. For example, V2X equipped traffic lights can inform drivers about the time until the next traffic signal change or indicate the optimum speed to pass the intersection with green light [1,2].

During the last years, the focus in cooperative ITS systems based on V2X has been in research and the specification of technical requirements. The standardization of requirements will be finalized in the near future. However some challenges related to legal issues, overall security system and definition of common performance criteria for V2X equipment still have to be met. Standards are only one important prerequisite for the deployment and market introduction of V2X [1].

Application level standard protocols for cooperative ITS define some important applications, their services and message transmission sequences. These predefined applications are generally called use cases. The services and message sequence of an ITS application protocol are fixed and applications should implement a rigid-formatted message set and message sequences for each use case [2].

As Cooperative ITS use cases create newly, it should be specified the protocol which is designed to be flexible and extendable, which enables to add or delete use cases conveniently. In this paper, we provide an overview on a standardization activity in ISO TC204 WG17 for ISO 13184 series. The ISO 13184 specifies the road guidance use cases on Data eXchange Messages (DXMessage) to provide the real-time decision support system to drivers or pedestrians using nomadic and portable devices (smartphones). The road guidance protocol (RGP) is an instantiation of the DXM, which represents a generic message to exchange data between ITS stations. The DXMessage contents are independent on the implemented use cases [2].

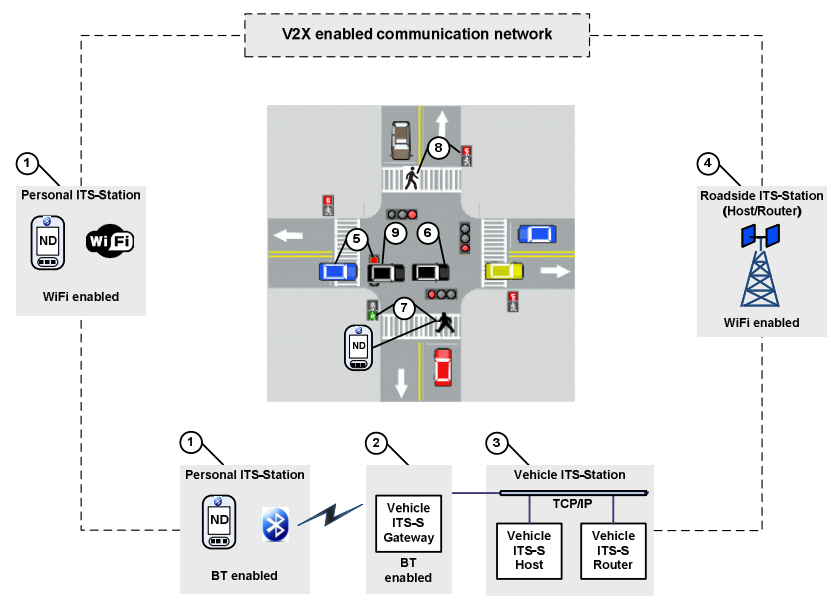

Figure 1. Overview of crossroads safety warning guidance[1]

Figure 1 shows an overview of crossroads safety warning guidance which is one of use cases on the RGP. In the figure, Personal ITS station (1) equipped vehicle approaching intersection receives message about the intersection geometry, status of the traffic signal and position correction information from the Roadside ITS Station(4)). In the vehicle, Personal ITS station connect to Vehicle ITS-Station(3) through Vehicle ITS-Station Gateway(2)). In ISO 13184-1, several use cases are defined such as violation a stop signal or traffic 
signal without stopping by vehicle or pedestrians (5), (6), (9)), Guiding the pedestrian on a pedestrian crossing(7), and bumper to bumper traffic conditions on the crossroads(8)). In ISO 13185-2, it specifies the flexible and extendible protocol between Personal ITS station (P-ITS-S)/Vehicle ITS station (V-ITS-S) and Roadside ITS station (R-ITS-S).

The remainder of this paper is organized as follows: First, we survey the related work, present motivation and important considerations for V2X-based safety applications in section 2 . In section 3, we designed the road guidance protocol (RGP) from the important considerations. Afterwards, we give a short description of our field test for RGP applied Cooperative Autonomous Vehicle Guidance system at cross roads in section 4. Finally, we conclude our paper with an outlook how we see the project evolving in chapter 5 .

\section{RELATED WORK}

This section presents motivation and important requirements for a RGP applied Intersection Collision Warning Application (ICWA). As introduced earlier, there have been many projects for intersection collision warning application as a V2X application. In United States, the Vehicle Safety Communication 2 Consortium executed the CICAS-V (cooperative intersection collision avoidance system for violations) Project. The CICAS-V project was a four-year project to develop a cooperative intersection collision avoidance system to assist drivers in avoiding crashes in the intersection by warning the driver of an impending violation of a traffic signal or a stop. Members of VSC2 are Ford Motor Company, General Motors Corporation, Honda and so on. Any Vehicle ITS station conforming to this CICAS will be receiving and decoding the SPaT messages which are Signal Phase and Timing Messages containing some Intersection topological data elements [5].

V2X-based road safety applications have different purposes from providing information and served by CAM (Cooperative Aware Message), DENM (Decentralised Environmental Notification Message) transmission and SPaT messages [4]. The existing services and messages sequence of V2X-based road safety applications are fixed and applications should implement a rigid-formatted message set and message sequences for each use case. V2X-based road safety applications can be created providing information about road hazard, signals, and stop sign, etc. These newly added applications need the protocol which is designed to be flexible and extendable, which enables to add or delete use cases conveniently.

In many cases, the applications for Cooperative ITS are designed to provide a set of messages that is dependent on the use cases and the message exchange method. However it is not easy to enumerate all use cases. The use cases can be changed or enhanced frequently. For Cooperative ITS application, the existing protocol designed depending on the use cases, is not appropriate. Therefore, we designed the standard protocol of ISO 13184 series which provide a general technique of designing the road guidance application protocol based on the Cooperative ITS use cases.

\section{III.RoAd Guidance Protocol}

The road guidance protocol (RGP) is an instantiation of the data eXchange message (DXMessage), which represents a generic message to exchange data between ITS stations. The RGP defines an interoperable service protocol between P-ITSS/V-ITS-S and R-ITS-S for exchanging data elements.

Figure 2 shows the real-time RGP communication of the road guidance implementation using the DXMessage. The PITS-S starts with "App" handling only messages from and to the R-ITS-Ss. The P-ITS-S is pre-loaded with the DXMessage configuration using case(s)-specific configuration information from the Center ITS station (C-ITS-S) to support road guidance messages and data parameters. After a road user (vehicle, pedestrian) enters a road guidance zone of an R-ITS$\mathrm{S}$, the P-ITS-S immediately reports its position and motion parameters with the necessary message to the R-ITS-S. If a situation occurs in the road guidance zone, which matches a supported use case, the R-ITS-S notifies the situation (i.e., safety warning, parking guidance) information to the P-ITS-S. The P-ITS-S shows the situation on the display and / or does some acoustic signals. On leaving the road guidance zone the P-ITS-S stops the communication to the R-ITS-S. Independent from the P-ITS-S to C-ITS-S / R-ITS-S communication, new messages and their parameters have to be communicated from the C-ITS-Ss to all R-ITS-Ss. In addition, the applications have to be modified and provided in the App Store to support all use cases $[2,3]$.

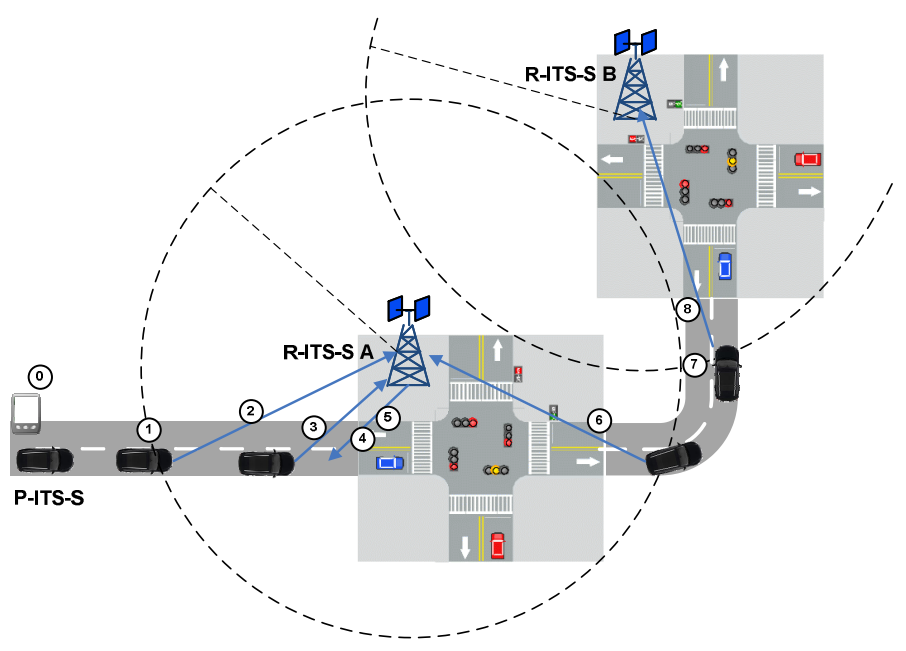

Figure 2. Road guidance protocol message exchange scenario [2]

In figure 2, the road guidance protocol message exchange sequence is as follows:

๑ RGP App configuration or configuration update

(1) Entering Road guidance zone of R-ITS-S A

(2) (3) Sending 'notify-on-position' containing position, motion parameter and vehicle size to R-ITS-S A 
(4) Getting 'collision-possible' from R-ITS-S

(5) Getting 'vehicle-blocks-road' from R-ITS-S

(6) Sending 'stop-notify' and leaving road guidance zone

(7) Entering road guidance zone of R-ITS-S B

(8) Sending 'notify-on-position' containing position, motion parameter and vehicle size to R-ITS-S B

After starting the road guidance app the configuration is performed. The vehicle is driving on the road. After entering the road guidance zone (1) of R-ITS-S 'A' it sends its current position, motion parameter and vehicle size to the R-ITS-S 'A' (2). The vehicle goes on driving and sends it current position again to R-ITS-S 'A' (3). R-ITS-S 'A' recognizes a problem with another vehicle and sends a 'collision-possible' to the PITS-S (4)). Short time later a 'vehicle-blocks-road' notification will be sent (5). While leaving the Road guidance zone the PITS-S sends a 'stop-notify' (6). The vehicle goes on driving and enters another road guidance zone (7) and sends a 'notify-on-position' to R-ITS-S 'B'. Figure 3 shows the RGP real-time message flow using the same numbers as used in figure 2 .

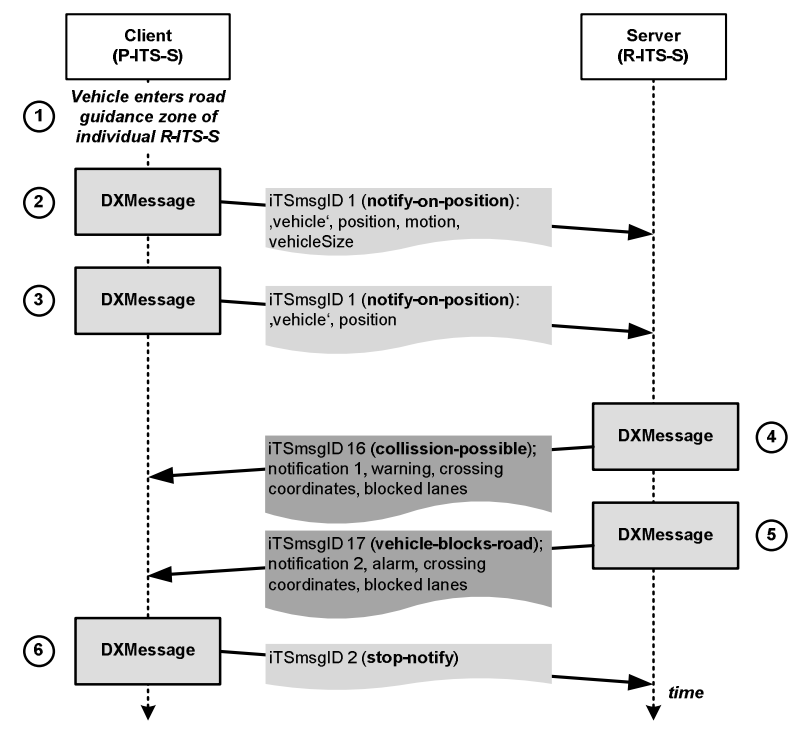

Figure 3. RGP real-time message flow [2]

A DXMessage is the ASN.1 structured container for ITS PDUs communicated between ITS stations. The DXMessage is encoded in unaligned Packed Encoding Rules (U-PER). The DXMessage was defined as follows.

\section{A. DSM ASN.1 definition}

DataExchangeMessage $\{$ iso(1)standard(0)dxm(13184)message(2)version(1)\}

DEFINITIONS AUTOMATIC TAGS ::= BEGIN
EXPORTS dxmVersion, DXMessage;

IMPORTS UNUM32, DataParamValue, Identifier, Version

FROM VehicleInterfaceDataFormat \{iso(1) standard(0) ugp(13185) vidf(0) version1(1) \};

dxmVersion Version $::=1$

DXMessage ::= SEQUENCE \{

version Version,

iTSmsID Identifier,

time UNUM32 OPTIONAL,

value SEQUENCE OF DataParamValue,

dtcInfo SEQUENCE OF DtcInfo OPTIONAL,

\}

END

- version - version of the DXMessages

- iTSmsID - ITS message identifier (see ISO/TS 17419)

- time- the time stamp of the DXM in milliseconds

- value - List of data parameter values

\section{B. DXMessage Example}

Table 1 shows an example for notify-on-position which is containing the road user type, the GPS position of the vehicle, the speed. The road user type is 'vehicle'. The GPS position of the vehicle is $51.06549^{\circ} \mathrm{N}, 6.09781^{\circ} \mathrm{E}, 166.8 \mathrm{~m}$. The speed of vehicle is $19.44 \mathrm{~m} / \mathrm{s}$ driving to the east $\left(45^{\circ}\right)$. The vehicle has the size has the size $4.48 \mathrm{~m} \times 1.78 \mathrm{~m} \times 1.57 \mathrm{~m}(\mathrm{~L} \times \mathrm{W} \times \mathrm{H})$ and a mass of $1450 \mathrm{~kg}$.

TABLE 1. EXAMPLE FOR SENDNOTIFYONPOSITION[2]

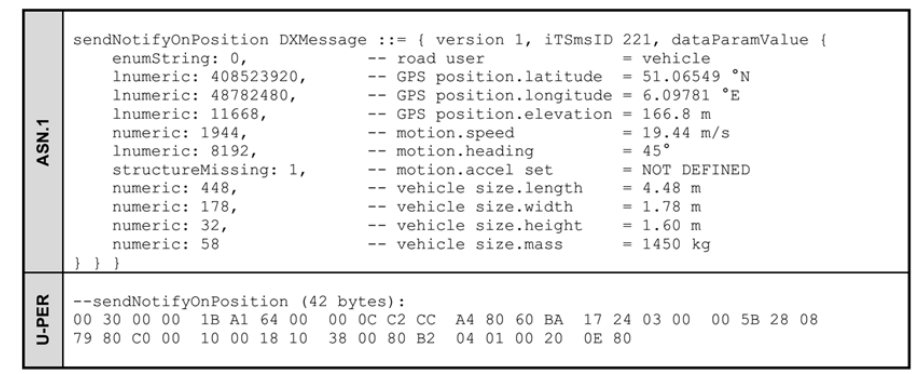

IV.FIELD TRIAL

We had a field trial of the RGP applied Cooperative Autonomous Vehicle Guidance (CAVG) system at a threelegged intersection as a test site. The CAVG system autonomously guides vehicles from a starting point to a destination within a local service area. The CAVG system is composed of several subsystems as follows [6]:

- Cooperative Vehicle Control Subsystem: CVCS

- Vehicle Guidance Communication Subsystem: VGCS

- Vehicle Guidance Terminal Subsystem: VGTS

- Infrastructure-based Recognition Subsystem: IRS

- Vehicle Guidance Server Subsystem: VGSS

The VGTS is a P-ITS-S/V-ITS-S which shows the situation or safety related information to drivers or pedestrians. The IRS fuses two heterogeneous sensors, a camera and a laser scanner. The laser scanner estimates distance to objects within a local service area. The camera identifies types (vehicle, 
pedestrian and obstacle) of objects [6]. The Vehicle Guidance Server Subsystem has monitoring functions and the decision algorithm for priority at the three-legged intersection based GPS and map data from vehicles within services area. The VGSS detect potential vehicle collisions at intersection. Each vehicle periodically exchanges location information (e.g., position, moving speed, direction and so on) with the VGSS using WAVE/DSRC technology. Figure 4 show the webbased VGSS.

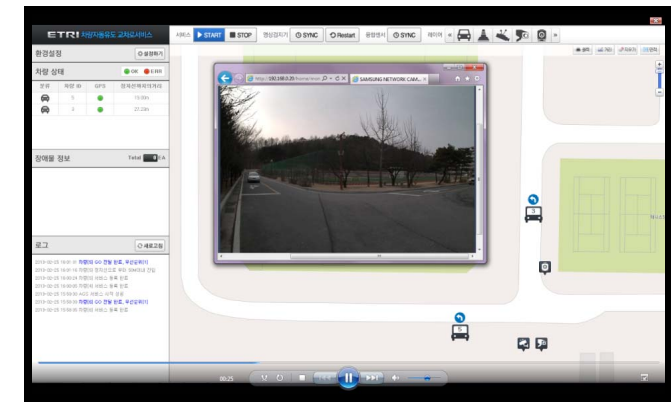

Figure 4. Autonomous vehicle guidance server subsystem

The VGSS sent service advertising messages periodically using ITSmsgID 17 (service-advertising). The VGTS register and report vehicle status periodically (every $500 \mathrm{msec}$ ) using ITSmsgID 1 (notify-on-position). The VGSS determines whether a vehicle will "Go" and "Stop" at the intersection and sent the information included "Go or Stop" and obstacle information within intersection using ITSmsgID 16 (collisionpossible). The obstacle was detected by the IRS. Figure 5 shows the status of the CVCS(a), VGTS(c), and IRS(d) when the intersection is occupied by another car. Figure 6 shows the status of the $\operatorname{CVCS}(a), \operatorname{VGTS}(\mathrm{c})$, and IRS(d) when the intersection is no longer occupied.

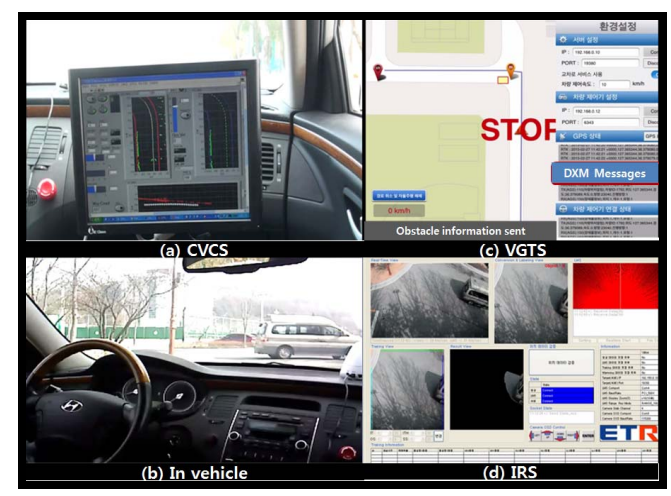

Figure 5. A crossroads intersection with two cars approching

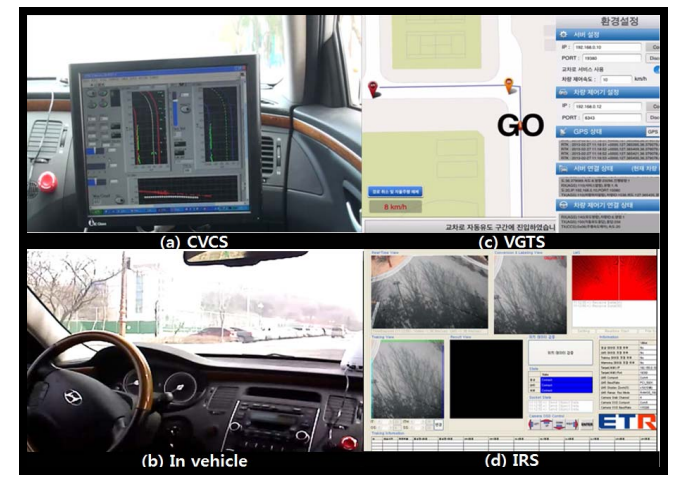

Figure 6. A crossroads intersection with only one car approaching

\section{Conclusions}

In this paper, we presented the main concept of the international standard protocol ISO 13184 series which provide a general technique of designing the road guidance application protocol based on the Cooperative ITS use cases. And also we presented the field trial of the RGP applied Cooperative Autonomous Vehicle Guidance (CAVG) system at a three-legged intersection as a test site. The ISO 13184 series allows flexibility and extendibility of the newly defined Cooperative ITS applications and this enables to add or delete use cases conveniently.

Finally, In Korea, Ministry of Land, Infrastructure and Transport has been built a long-term C-ITS deployment plan and selected 15 C-ITS services for the first C-ITS deployment such as a vehicle collision prevention support service, intersection collision prevention support services, and location-based data collection. Standard is one of the important preconditions for C-ITS deployment and market introduction. In particular, it is important for C-ITS technology export to have an active participation in International Standards Organization with national technical standards.

\section{ACKNOWLEDGMENT}

This research was supported by the ICT Standardization program of MISP (The Ministry of Science, ICT \& Future Planning).

\section{REFERENCES}

[1] C-ITS systems, KAPSH, 2008. Available: http://www.kapsh.net/

[2] Intelligent transport systems - Guidance protocol via personal ITS station for advisory safety systems - Part 1: General information and use case definitions, ISO TR 13184-1, 2013.

[3] Intelligent transport systems - Guidance protocol via personal ITS station for advisory safety systems - Part 2: Road Guidance Protocol (RGP) requirements and specification, ISO/NP 13184-2, 2013.

[4] Intelligent Transport System (ITS); V2V application; Part 2 Intersection Collision Risk Warning (ICRW) application specification, ETSI TS 101 539-2, 2013.

[5] CICAS-V Project Final Report, 2011. Available: http://www.nhtsa.gov

[6] D.Y. Kwak, "A Cooperative Autonomous Vehicle Guidance System," $19^{\text {th }}$ ITS World Congress, October 2012. 
[7] H. Oh, "Recent Trends on V2X Communication Technology in Europe," AUTO Journal, Vol.35, No.11, pp.20-26.

[8] Javier Alonso, Vicente Milanes, and Joshue Perez, "Autonomous vehicle control systems for safe crossroads," IEEE Trans. Research Part C, vol. 19, pp. 1095-1110, 2011.

Hyun Jeong Yun received her MS from CHONBUK National University of Computer engineering in 1999. She is currently a senior researcher at Electronics and Telecommunications Research Institute, Korea. Her research interest includes the in-vehicle network technology, autonomous vehicle systems and IP-based In-Car communication. Also she is a project leader for ISO 13185 series in ISO TC204 WG17.

Jeong Dan Choi received her $\mathrm{PhD}$ from Chung-Nam National University of Computer Science in 2005. She is currently a director of Car/infra fusion Research Team at ETRI, Korea. Her research interest includes the Computer Graphics and Image Processing technology, and autonomous vehicle systems. 